\title{
Localized Drug Delivery for Cardiothoracic Surgery
}

\author{
Christopher Rolfes, Stephen Howard, Ryan Goff and Paul A. laizzo
}

Additional information is available at the end of the chapter

http://dx.doi.org/10.5772/48577

\section{Introduction}

It is noteworthy to consider that extensive bioavailability and bioequivalence studies are typically required before new drug therapies can be approved [1]. These studies include pharmacokinetic studies that take into account: 1) dosing, absorption, and elimination rates of the drug and its active metabolites, as well as 2) the potential effects of multiple doses, drug interactions, and the differences whether medications are taken with or without food. A major therapeutic factor that compounds the variations often seen from patient to patient is individual differences in absorption and elimination rates. This will also cause variations in the amount of drug that reaches the desired targeted tissue when used as a clinical therapy.

While oral administrations are common and the easiest means to deliver outside of a hospital or clinical setting, intravenous (IV) delivery can eliminate some of the aforementioned patient to patient variability by bypassing the ingestion and absorption into a patient's bloodstream. However, a major obstacle with either of these delivery methods is that once a drug is in the blood plasma, the medication will circulate throughout the patient's body, not only reaching the intended site, but unintended sites as well. Hence, this will greatly increase the possibility of causing unwanted side effects. Thus, it is required that side effects on each and every tissue be well described when therapeutic levels of the medication are administered.

Importantly, many drugs have described narrow therapeutic ranges. Slight increases in levels could cause severe undesired effects, whereas slight decreases often eliminate any therapeutic benefit. We describe in detail here how the targeted and local delivery of medications may overcome many of these obstacles in traditional delivery methods by simplifying the pharmacokinetics, reducing variability, and allowing higher doses to reach the intended target. 


\subsection{Targeted drug delivery}

"Targeted drug delivery" is a general term that describes a variety of methods that can be used to increase the concentrations of a given drug at a primary location within the body relative to other body tissues. Often called "smart therapies," targeted delivery includes methods such as antibody labeling, ultrasonic release, and/or localized delivery that can increase drug concentrations at the desired tissue. The primary intent is to increase the intended beneficial effects while reducing side effects.

Developments in targeted drug delivery were commonly pioneered with anti-cancer drugs. These treatments are often highly toxic and have undesirable side effects, which in turn can greatly reduce quality of life and limit the dose levels that can be administered. Therefore, if the levels of these drugs can be increased specifically at the site of a tumor relative to the rest of the body, the same or reduced dose levels will have much greater effects at the site of the cancerous tumor.

One commonly described method for accomplishing this is creating or adding components to cancer drugs that preferentially bind within the tumors. More specifically, the identification of differences in endothelial surfaces in growing tumors has led to the development of cancer medications that preferentially adhere to the endothelial surfaces within the vasculature of the tumor, thus increasing the desired effects. This results in lower exposures of non-target tissues to the drug than were previously possible. In a similar manner, numerous biomarkers have been identified that become upregulated in diseased cardiac tissue, and therefore have become targets in emerging therapies [2], [3]. Alternatively, drug treatments can be encapsulated in such a way that they are released at the desired location, such as where triggered by high frequency ultrasound, causing focal increases in drug concentrations [4-6]. Table 1 summarizes many currently used and investigated targeted therapies, several of which are described in greater detail throughout this chapter.

\subsection{Localized delivery}

"Localized drug delivery" is defined as a specific form of targeted delivery where the medication is given at a certain site which allows for reduced movement and subsequent absorption into the bloodstream. Localized delivery is often provided to a naturally enclosed space, such as the bladder or into the vitreous humor of the eye, but other techniques can limit movement such as a gel or patch. In general, by delivering a given pharmacological treatment to a specific target tissue site via an implantable pump or acute access, localized therapy will reduce systemic effects on peripheral tissue thereby limiting side effects, while maintaining increased control.

Just as IV delivery increases control and decreases variability compared to oral delivery by eliminating the gastrointestinal tract, local delivery increases control and decreases variability by eliminating reliance on patient circulation for distribution. Thus localized drug delivery carries the potential for increased effectiveness of treatments, while reducing 
the quantities needed (Figure 1). These reductions have important applied implications when one employs either drug pumps or impregnated gels to delivery therapies, as they can only hold limited volumes.

\begin{tabular}{|l|l|l|}
\hline \multicolumn{2}{|l|}{ Delivery Method } & \multicolumn{2}{l|}{ Disadvantages } \\
\hline $\begin{array}{l}\text { Targeted Drug Delivery } \\
\text { carriers }\end{array}$ & $\begin{array}{l}\text { non-invasive focal } \\
\text { treatment to potential } \\
\text { asymmetric areas }\end{array}$ & $\begin{array}{l}\text { equipment intensive, } \\
\text { potential buildup within liver } \\
\text { and spleen }\end{array}$ \\
\hline Biomarker targeted & simple administration & $\begin{array}{l}\text { designer molecules need to } \\
\text { be created, approved }\end{array}$ \\
\hline Localized Drug Delivery & $\begin{array}{l}\text { entire epicardium treated, } \\
\text { well contained, easy access } \\
\text { in surgery }\end{array}$ & $\begin{array}{l}\text { invasive, pericardium often } \\
\text { left open after surgery }\end{array}$ \\
\hline Pericardial delivery & $\begin{array}{l}\text { increases myocardial } \\
\text { concentrations, long lasting }\end{array}$ & invasive/minimally invasive \\
\hline $\begin{array}{l}\text { Direct myocardial/tissue } \\
\text { injection }\end{array}$ & $\begin{array}{l}\text { long or short lasting, } \\
\text { tunable degradation }\end{array}$ & $\begin{array}{l}\text { minimal migration small } \\
\text { doses, reliant on resorbable } \\
\text { wafer or must be explanted }\end{array}$ \\
\hline Drug eluting wafers & $\begin{array}{l}\text { local drug delivery on } \\
\text { demand, larger continuous } \\
\text { dosing possible }\end{array}$ & $\begin{array}{l}\text { invasive, needs refilling, } \\
\text { shortcomings associated with } \\
\text { implantable devices }\end{array}$ \\
\hline implantable pump & $\begin{array}{l}\text { increases myocardial } \\
\text { concentrations } \\
\text { treatment still enters blood }\end{array}$ \\
\hline Coronary injection
\end{tabular}

Table 1. Various targeted therapies and their advantages and disadvantages.

Finally, an additional benefit often seen with localized delivery is a relative increase in the therapeutic drug half-lives. For example, since localized treatments typically have minimal crossover with the patients' circulation, there is limited exposure to their livers and kidneys, which are typical sites for drug metabolism. Thus the relative therapeutic half-lives of many pharmaceuticals will be increased, creating another mechanism to decrease the amount of a given agent required to achieve sustained therapeutic dose levels [7].

While targeted drug delivery is the focus of broad research, it is our intent with this chapter to narrow the focus more specifically on localized therapy and the unique opportunities provided by the access obtained during thoracic surgery. For instance the pericardium surrounds the heart and provides a unique enclosed volume in which one can target the epicardial surfaces. In other words, the localized drug delivery to the pericardial space will allow the agent to diffuse into the myocardium while reducing those amounts present in the circulating blood. During cardiothoracic surgery one has unique access to this otherwise difficult to reach space where subsequent therapy can be delivered throughout the perioperative period. Therefore, as therapies emerge to treat heart failure, local delivery 
during thoracic surgery is positioned to be a viable therapeutic option that can be delivered with minimal added time, as well as few complications or complexities. At the same time it has the potential to reduce ischemic damage and arrhythmias, and holds great potential for improved outcomes, reduced morbidity, and increased cardiac health.

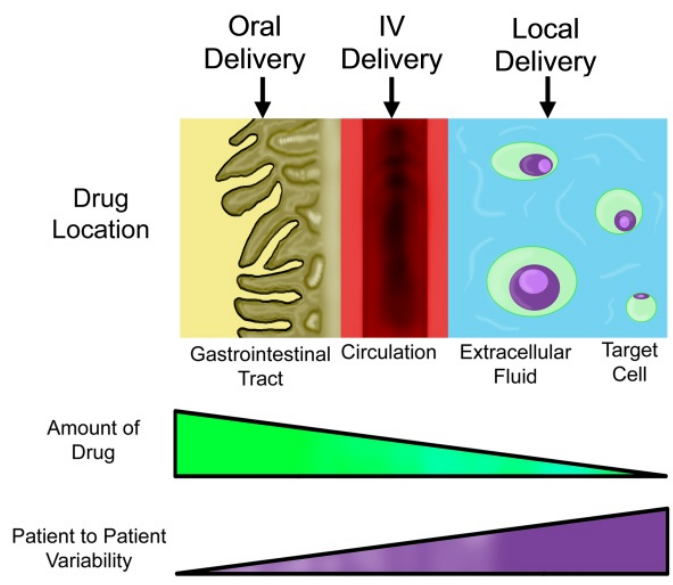

Figure 1. Schematic of differences in drug delivery by oral, IV or local delivery modalities. Increasing the number of transport steps increases the amount of drug necessary and patient to patient variability increases at each step.

\section{Known procedural complications and potential therapeutic opportunities}

Cardiac surgery is typically defined by a broad class of surgeries intended to correct heart problems. They can be broken down into several categories that include: 1) valve repair and replacements, 2) structural heart repairs, 3) implantations of devices for either the maintenance of rhythm or mechanical performance, and 4) heart transplants. Today, the most common cardiac surgery is coronary artery bypass grafting (CABG) [8]. Despite the recent trend towards minimally invasive cardiac procedures, there still remains a significant need for open surgical techniques. Due to the invasive, and many times emergent, nature of these procedures they are also excellent candidates for targeted delivery (it should also be noted that many minimally invasive and scheduled procedures could also take advantage of target therapeutic delivery of various agents).

It is generally accepted that open cardiac surgery is not without potential complications. On the other hand, the occurrences of such complications can be viewed as unique opportunities for introduction of localized drug delivery to improve patient outcomes. It is important to note that complication rates and outcomes can only be truly assessed on both procedural and patient population bases; yet there are often common complications across all classes of cardiac surgery. Statistics on the following procedural complications and proposed treatments using targeted delivery will be discussed: neurologic, arrhythmic, 
gastrointestinal, bleeding, and infection. These complications when present will impact both short- and long-term morbidity and mortality, i.e., influence length of hospital stays, quality of life, as well as financial burdens on both patients and healthcare providers.

To begin with, neurologic complications such as stroke, post-operative cognitive deficit, encephalopathy, and transient ischemic attack are of particular concern because of their much feared, potential long-lasting effects. Due to the nature of cardiac surgery and cardiopulmonary bypass, it can be foreseen that thrombosis or air emboli may occur from time to time, potentially causing these aforementioned complications. Poor neurological outcomes may also be caused by or related to arrhythmias, bleeding, or other complications. For example, prospective studies on the outcome of CABG patients show that the expected stroke rates vary from $1.5 \%$ [9] to 5.2\% [8]. A broad range of disorders fall under the umbrella of post-surgical encephalopathy, a syndrome stemming from many causes. Its prevalence has been described to vary based on the definition, with as many as one third of patients eliciting an encephalopathy post-operatively [10], but prospective studies show that by day four the number is reduced to $11.6 \%$ [11] indicating a transient nature. Expectedly, these complications occur more frequently in combined procedures and/or more technically challenging cases.

It should be mentioned that numerous modern surgical techniques, such as the no-touch aorta technique, have attempted to mitigate such neurologic complications. However, it will likely remain that current advances in surgical techniques may only reduce, but not totally eliminate, these undesired events. Nevertheless, one can envision the added use of tailored drug cocktails with various procedures that could directly target the heart (pericardial space) or mix within the blood returning from cardiopulmonary bypass. To date, potential therapeutic agents which have been shown to be effective include: 1) oxide scavengers, 2) nitric oxide inhibitors [12], 3) agents to inhibit glutamate related neural excitotoxicity [13], and 4) the administration of aprotinin, a serine protease inhibitor [14]. It is possible that prior to patient rewarming, agents similar to tissue plasminogen activator could be administered in a manner that targets or localizes only the cerebral circulation and potentially destroys clots that were formed, thus mitigating negative effects prior to rewarming.

It is generally considered that atrial fibrillation (AF) is the most common complication to occur after cardiac surgery, occurring in up to $30 \%$ of cases [15]. A recent meta-analysis of 94 trials by Burgess and colleagues has shown that the five most common interventions were all effective at reducing the incidence of $\mathrm{AF}$, but to varying degrees. These treatments included beta-blockers, sotalol, amiodarone, magnesium, and atrial pacing. It should be noted that amiodarone was the only intervention that was reported to reduce stroke on its own. Nevertheless due to the well documented negative systemic effects of such antiarrhythmics, several studies have begun to investigate the use of localized delivery to the pericardial space, discussed in more detail below. On the other hand, it should be noted that the systemic administration of modified agents could also prove effective and avoid side effects if targeting functionality was incorporated into the drug molecule. For example, 
this could be done by adding cardiac specific antibodies or ligands to therapeutic molecules. Nevertheless, such newly developed drug isoforms will require additional regulatory approval, perhaps only after clinical studies prove them safe and effective.

Gastrointestinal complications after cardiac surgery are less common, but importantly are associated with high rates of morbidity and mortality. Reported incidences are in range of $<1 \%$, but mortality associated with this complication is approximately $25 \%$ versus approximately $3 \%$ without gastrointestinal complications [16], [17]. The most common of these complications include: upper intestinal bleeding, intestinal ischemia, acute pancreatitis, and perforations.

Despite the use of modern day antibiotics, infection still remains a prevalent problem in cardiac surgery, as does administration of blood products. The two are commonly interrelated and it has been shown that transfusions of packed red blood cells (PRBCs) are significantly associated with post-operative infections [18]. However, this correlation does not necessarily imply causation, and the use of PRBCs may be related to procedural difficulties, which may be the real underlying causation of such infection rates. It should also be considered that stored red blood cells also do not function normally, and therefore PRBCs may contain inflammatory and immuno-mediating substances. It was reported in a CABG patient series study at Duke University that as many as $39.5 \%$ of patients received PRBCs [18]. Furthermore, out of the entire series, the post-operative infection rate was found to be $6.2 \%$; specifically, patients that received PRBCs elicited infection rates ranging from 6$8.1 \%$ versus $5.1 \%$ for those who received no PRBCs [18]. Additionally, in a recent five-year study on long-term survival following transfusion for CABG procedures, it was observed that patients not transfused have approximately 2.5-fold better survival rates than those that did [19].

Interestingly, it has been proposed that actively targeted treatments could possibly restore normality to the RBCs prior to administration; such treatments could also incorporate antiinfection components. As mentioned above, RBCs can change drastically and immediately upon storage. It is considered that due to the low oxygen environment within the blood collection bag, RBCs switch to anaerobic metabolism, which in turn leads to lactic acid buildup and an overall reduction in blood $\mathrm{pH}$. Furthermore, it is known the RBCs lose their signature bi-concave shape and become more spherical with storage; recall that it is this biconcavity that is necessary for the cells to efficiently travel through capillary networks. Stored RBCs will also form aggregates due to activated surface proteins by crosslinking fibrinogen between GPIIb/IIIa binding sites [20]. This crosslinking increases the longer PRBCs are stored, and potentially could contribute to neurologic complications and thus post-operative cognitive deficits. Therefore, one could consider that prior to infusion of PRBCs, a targeted drug cocktail could be added to prevent or break these fibrinogen crosslinks and/or suppress immuno- and inflammatory effects.

Finally, one should also consider that infection rates could potentially be reduced by localized drug delivery at the end of a given cardiothoracic surgical procedure. For example, one such method might take the form of an antibiotic that could be sprayed on or within the 
thoracic cavity immediately prior to and after closure of the surgical entrance site. Such an application method may also have the potential to reach interstitial areas that are at risk for infections, specifically those with inherently minimal blood flows; in other words, these tissue areas would otherwise receive minimal amounts of orally or intravenously administered antibiotics.

\subsection{Other drug targets}

With recent advances in microfabrication, it is feasible to locally deliver drugs in ways that were previously not possible. The field capsule endoscopy is a good example of these technologies and also one that may be further miniaturized and exploited for drug delivery. "Capsule endoscopy" is the swallowing of a pill with a video camera inside. The pill travels through the gastrointestinal system and records its journey. Even more sophisticated versions of these devices are being developed to be actively propelled by magnetic energy [21] or flagella type [22] propellers. It should also be noted that magnetic pills for drug delivery are currently under development [23]. One should consider that these novel technologies may also be exploited for cardiac use. For example, a patient could have a magnetic pill guided endovascularly to the heart and anchored to the endocardial surface where therapeutic (biologics or drugs) agents are released. Furthermore, one could even envision that the administration of these therapeutics could be controlled wirelessly and facilitated by micropumps and valves.

In the near future, it is considered that drug eluting microfabricated devices with incorporated biosensors could be implanted locally such that they release drugs in response to given physiological stimuli. For example, a small sphere, capsule, or micelle containing insulin producing cells could be implanted in the pancreas, subdermally, or intramuscularly. The cells could then respond to fluctuations in glucose levels automatically. Extensive research has gone into such closed loop systems for insulin delivery for diabetes patients, with the eventual goal of developing an artificial pancreas [24-27]. In the more distant future, genetically engineered cells could be programmed to produce other drugs as needed and subsequently deliver them at the proper rates or in response to particular stimuli. Furthermore, unlike implantable devices such as drug eluting stents that can only deliver drugs for a few years, these cell-based drug producing devices have the potential to last a lifetime.

It is generally considered that delivering therapeutic agents at or near the entrance to coronary arteries would be particularly useful in certain clinical situations. Such delivery methods could then exploit the natural capillary system to perfuse the drug to the entire heart. This could in turn potentially reduce the amount of drugs needed significantly and may also ameliorate undesired side effects known for systemic administration. In another approach, during surgical operations, deployed degradable microcapsules with tuned drug release profiles could be injected into the myocardium adjacent to the coronary arteries. Alternatively, resorbable patches could be adhered over the main coronary arteries and the therapeutic agent would then diffuse into the vessel and be transported to the entire organ. 
In addition, a delivery patch could also be adhered to local areas, such as one doped with angiogenic treatment placed on an infarct zone.

In the future, combinatorial therapies could also be extended beyond current clinical practice such as drug eluting stents and pacing leads. This is an area of great opportunity for local drug delivery advancements. For example, ventricular assist devices could incorporate the ability to passively or actively deliver a variety of therapies. As such, their possible approaches and advantages may improve the use of these devices, e.g., when they are being used as a bridge to transplant or recovery.

It should be noted that the direct injection of drugs, proteins, or cells into the myocardium has also been proposed as a method of local delivery [28-31]. More specifically, these could be localized in areas of infarct or near atherosclerotic plaque deposits to aid in restoration of normal function. To date, it is noteworthy that positive results have been observed with injections of adenoviruses encoding for heat shock protein [28] or growth hormone [29], [30] in rabbit and rat models, respectively. Furthermore, clinically, gene transfer by direct injection of plasmid DNA coding vascular endothelial growth factor (VEGF) into ischemic myocardium has promoted angiogenesis [31].

It should be described for completeness that transmyocardial laser revascularization has been applied clinically to patients with inoperable coronary artery disease and often used in conjunction with $\mathrm{CABG}$. This procedure utilizes a laser to perforate the walls of the heart in areas of poor perfusion. The channels created act as conduit for blood and, during healing neovascularization, and also considered to improve perfusion. Perhaps this approach, if considered an option, could be supplemented with adjuvant use of local therapeutic agents to quicken the vascularization process, such as VEGF, or other therapeutics to potentially restore normal function.

\subsection{Alternative local delivery methods}

To date, numerous drug pumps and other devices and methods for delivering treatment to a localized area or region of the body have been shown to be successful. As mentioned previously, there is a considered difference between local delivery and targeted delivery of treatments. Additionally, it is also possible that various therapeutic approaches incorporate one or both of these methods, in order to maximize the beneficial effects of the therapy and minimize the adverse side effects.

One such method, which has been studied significantly, is to create a polymer or biological scaffold in which the drug/protein has been embedded. Subsequent release is then dependent upon either degradation of the scaffold or diffusion of the drug out of the scaffold. Currently there are clinical devices available, such as the Gliadel ${ }^{\circledR}$ wafer, which is impregnated with a chemotherapy drug and used following surgical resection of cancerous tissue within the brain [32], [33]. Note that these drug delivery platforms have been made from a number of different polymers, synthetic or natural. Ultimately it is considered that whichever material is used, it must be biocompatible and able to dispense the drug at 
appropriate rates for the particular treatment. Currently, such products have been developed relative to treatments for cancers, which have included polymer structures for subcutaneous or intramuscular placement. More recently, similar scaffolds have been created for the treatment of cardiac diseases, but these are still within the research phase of the design.

Myocardial patches, often made of a gel, collagen, or other biocompatible material, are typically impregnated with stem cells or protein growth factors meant to diffuse into the heart to promote myocardial growth and revascularization. These are placed locally on infarcted areas of the heart. Alternatively, the scaffolds could be designed to promote growth within their structure, becoming a functioning part of the myocardium. If the device/scaffold is made such that it requires stem cell infiltration in an in vitro setting, it can be incubated with the particular cells needed prior to implantation onto the cardiac structure [34]. Both of these methods illustrate how tissue engineering approaches could be utilized to locally deliver drugs or therapies to the heart, however there are other mechanical devices that also allow a physician to deliver drugs directly to the site of interest.

\subsection{Localized injections and drug pumps}

When discussing localized treatment of tissue, a method to deliver the treatment to a specific region of interest is essential. Various methods have been reported in the literature, from simplistic methods of direct injections of the drug to the localized area to be treated to the use of more complex microelectromechanical systems (MEMS). The method for direct injection of a treatment into a specific diseased area is a fairly simplistic idea; however, the means to deliver a needle and treatment to the heart may pose a significant challenge. Current research is investigating injections into the myocardium via angioplasty balloons to reduce the occurrence of restenosis. More specifically, small pores or openings within a catheter deliver gene therapy or alternative drug treatments to the diseased cardiac cells [35], [36]. Likewise a given drug can be coated directly onto the exterior surfaces of a balloon, i.e., when the angioplasty is performed, the coating rubs off on the wall of the vessel to provide therapy. Recently, Scheller and colleagues demonstrated that such a device was able to decrease the incidence of restenosis significantly [37]. Stents themselves can also be thought of as a method for localized delivery of a drug to a specific location. This approach has been well developed and tested; drugs like paclitaxel have been coated onto the outside of a coronary stent to minimize restenosis that can occur at sites of stent implantation [38].

Other treatments that might be administered to a patient may need to be localized, but cover a greater area than a single location along an artery or vein. For those purposes, devices such as MEMS or osmotic pumps could perhaps be utilized to slowly deliver treatment to a specific site continuously or intermittently and with varying rates; i.e., delivery could last from days to months. When considering drug pumps, they generally fall into one of two categories, passive or active. The passive pump approach can be thought of as being similar to the gels or wafers discussed previously; however instead of the drug being embedded 
within the polymer, it would be contained within a chamber that would be opened once the polymer had been degraded enough to release it. To date, several designs have been implemented including multiple wells in a row with differing polymer closures to release at different times, as well as multiple chambers to release two different types of drugs simultaneously. As one could imagine, the only limitations to these types of designs are the properties of the polymers and the size of the device [32], [39].

One specific design described as a passive system is fairly unique- the use of osmotic pressure to push out the drug from a syringe-like device. These work on the principle that water will diffuse one way across a semipermeable membrane and increase the pressure on one side, thereby pushing out the drug slowly [39]. These types of devices have also been modified slightly to create pulsatile drug delivery pumps, i.e., in one case, a membrane was set up and had immobilized glucose oxidase which converted glucose to gluconic acid [40]. This results in an ionic change in the membrane, and the electrostatic repulsion of the membrane causes an expansion and increased delivery of insulin. This has the added ability to adapt to the specific needs of patients at various times of the day, depending upon glucose levels within their systems [40].

Another type of the MEMS approach for agent delivery is to release a bolus from a small reservoir; initially these devices relied on an electrochemical dissolution of a gold membrane blocking a reservoir of a solution. However, a number of published studies to date have reported that this approach could not be performed reliably, so it was modified to a localized melting of the gold membranes by resistive heating [32]. Another approach of an active delivery pump has been employed for patients with chronic pain, e.g., a pump with a reservoir filled with a pain medication can be implanted with a catheter leading directly into the spinal column or other neuronal targets. It should be noted that more recently the ability to refill these devices has been greatly improved and this is an advantage over MEMS devices that cannot refill, however the size of the former devices are currently much greater [41]. These types of pump systems have their own specific purposes, yet both intend to deliver a drug treatment to a localized area within the body to help reduce the effects of the drug on other organs or nearby healthy tissue.

\subsection{Ultrasound and lipisomal delivery approaches}

Another delivery technique is encapsulating agents for release in specific areas. As opposed to the local delivery pumps described above, where a drug is released into a specific location within the body, generally these packaged therapies can be administered intravenously with targeted release. In these cases, the drug will circulate throughout the entire body, but importantly the targeted drugs have been altered in a way to make them: 1) released at a specific site, 2) preferentially bind within the diseased area, 3) be more readily taken up by the target cells, 4) preferentially released slowly over time, or 5) any combination of these attributes. Ultimately the aim is to increase the effectiveness of the treatment while decreasing the toxic systemic effects of the drug. These approaches can be considered compatible with localized delivery, especially in the setting of thoracic surgery. For instance, 
a targeted drug could be infused into the coronary arteries, compounding the targeted design with local delivery.

Another specific way that investigators have been able to achieve targeted delivery is by using liposomes that encapsulate the drug, relying on active or passive targeting of tissues to be treated. First, it can act passively, relying on various cells to uptake the liposomes, e.g., reticuloendothelial cells, which can be found concentrated within the liver and spleen. Thus if the treatment was designed specifically for cells within the liver or spleen, a passive approach might be quite acceptable. It should be noted that this same approach has been discussed within nanoparticle delivery as well, taking advantage of the fact that the vasculature within tumors can be porous, allowing the nanoparticles to accumulate within the tumor region. It is still important, however, to aim to limit peripheral exposure, since such liposomes may still be taken up within the liver and other filtering organs [39], [42].

More specifically, an active targeting paradigm could be achieved by placing a recognition sequence on the outside of the liposome, to develop ligand-receptor interactions that will in turn bind the carrier to the targeted cells. As such, these could be targeted to specific proteins or to biomarkers that are upregulated in specific tissues, like tumors or portions of the myocardium responding to ischemia or heart failure [2], [3]. One needs to consider that these modified liposomes may still be taken up by the liver, spleen, or other non-targets; however, some specified modifications of the developed lipid layers may minimize uptake in these structures [42].

Another reported means that treatments may be delivered to target specific cells is via microbubbles aided by ultrasound disruption. More specifically, the microbubbles can be formed by air or other types of gas and introduced into the bloodstream similar to those techniques utilized in ultrasound imaging with contrast. Note that air bubbles are more readily dissolved into the blood following introduction into the venous system, giving them a shorter lifespan. By using perfluorocarbon gases, the lifespan of the delivery bubbles increase and they can also be coated with a variety of materials including polymers, lipids, or proteins which will further increase the lifespan. These longlasting microbubbles can then be disrupted by focused ultrasound - a trigger that can be applied nearly anywhere in the body [4-6]. It is considered that the ballistics of the cavitation not only disrupt the integrity of the bubble, but it will also momentarily disrupt the plasma membranes of the target cells, allowing for the drug and/or microbubbles to be passed through [43]. While this approach has resulted in detrimental effects on cardiac mechanical function [44], it has been shown to effectively increase absorption of certain drugs [43]. Alternatively, the capsules can be designed to release their therapeutic payload with a slight increase in temperature that can be triggered by local heating [45].

\section{Pericardial delivery}

It has been noted that the pericardium provides a unique space that holds a vast potential for localized drug delivery. For example, such pericardial approaches may range from: 1) 
the delivery of preconditioning therapies during surgical preparation, 2) providing for therapies that promote vascular genesis after $\mathrm{CABG}$, and 3) the prophylactic administration of antiarrhythmic agents in order to prevent post-operative AF. We believe that the possible treatments for the myocardium are numerous and will provide a few specific examples below, following a review of pericardial anatomy.

\subsection{Anatomy of the pericardium}

The pericardium is made up of two connected structures. The innermost layer is serous membrane which is inseparable from the epicardial surface, and is called the "visceral pericardium." The continuous serous membrane is folded in on itself and the single layer also makes up the inner surface of the parietal pericardium. The single layer of mesothelial cells is indistinguishable from the fibrous outer layer (Figure 2). Together, the parietal and fibrous layers make up the outer layer, or "parietal pericardium." This is the most prominent layer of the pericardium and is what we generally think of when we discuss the pericardium.

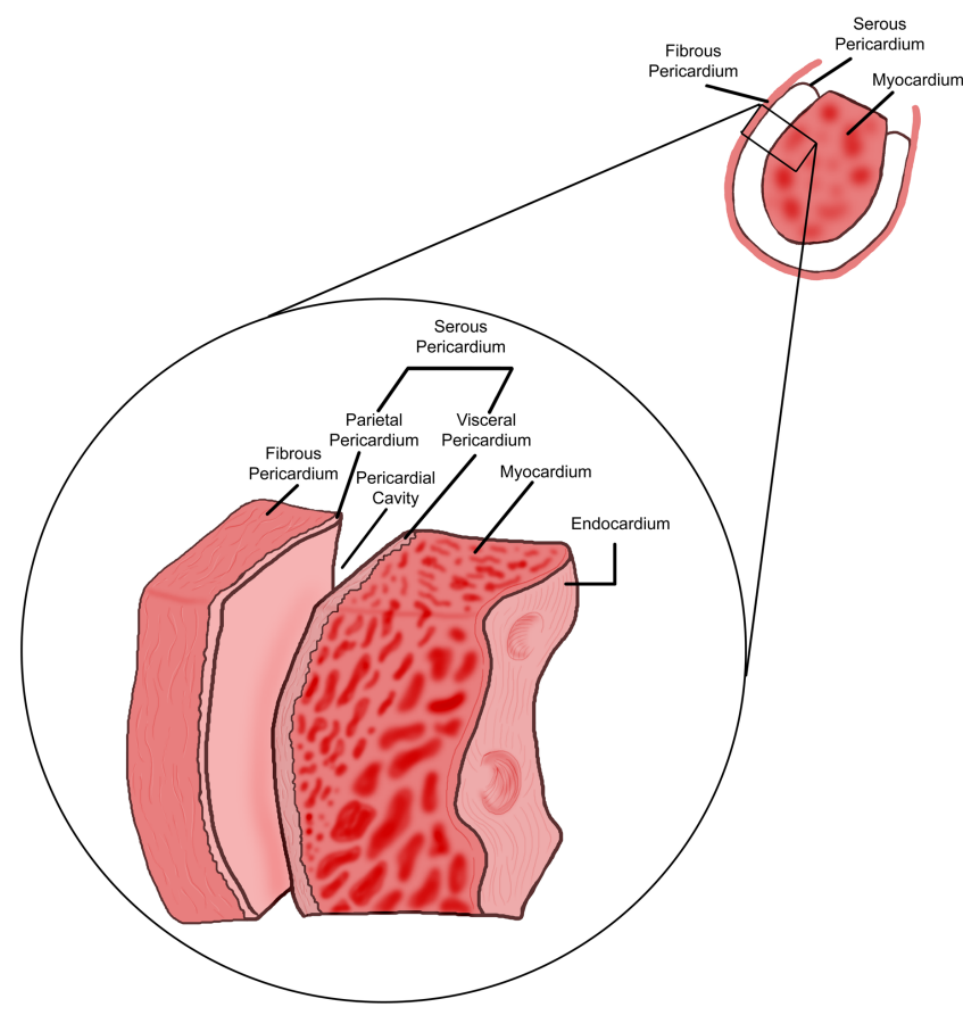

Figure 2. Schematic of the layers of the pericardium. The upper right shows a schematic diagram of the serous and fibrous pericardium with respect to the heart. The expanded cross-section view shows the attachment of two layers of the serous pericardium (visceral and parietal) to the myocardium and fibrous pericardium, respectively. 
The healthy pericardium contains $20-60 \mathrm{~mL}$ of pericardial fluid. This fluid, an ultrafiltrate of the plasma [46], surrounds the heart, with the majority concentrated in the pericardial sinuses and atrioventricular grooves. This fluid normally drains into the lymphatic system at a relatively slow rate, measured to be a volume equivalent to every 5-7 hours in sheep [47]. However, as pericardial fluid pressure increases, such as in the case of cardiac tamponade, investigators have found that not only does lymphatic drainage increase [48], but fluid may pass through the pericardium and enter the pleural space [49].

Though the volume of pericardial fluid is not evenly distributed, it is generally found to be well mixed due to the motion of the heart; thus agents can be considered to be quickly and evenly dispersed throughout [47]. Even though there is only a relatively small amount of fluid circulating around the ventricles, this aforementioned mixing action will help maintain even distribution of any additions to the pericardial fluid epicardially, thus maintaining consistent gradients relative to the myocardium. While the parietal pericardium is generally considered as non-compliant, the overall pericardial space can accommodate moderate increases in the amount of fluid by filling in the pericardial sinuses. However, once this reserve volume space is filled, pericardial pressure quickly increases with added volume, i.e., symptomatic tamponade is elicited (Figure 3).

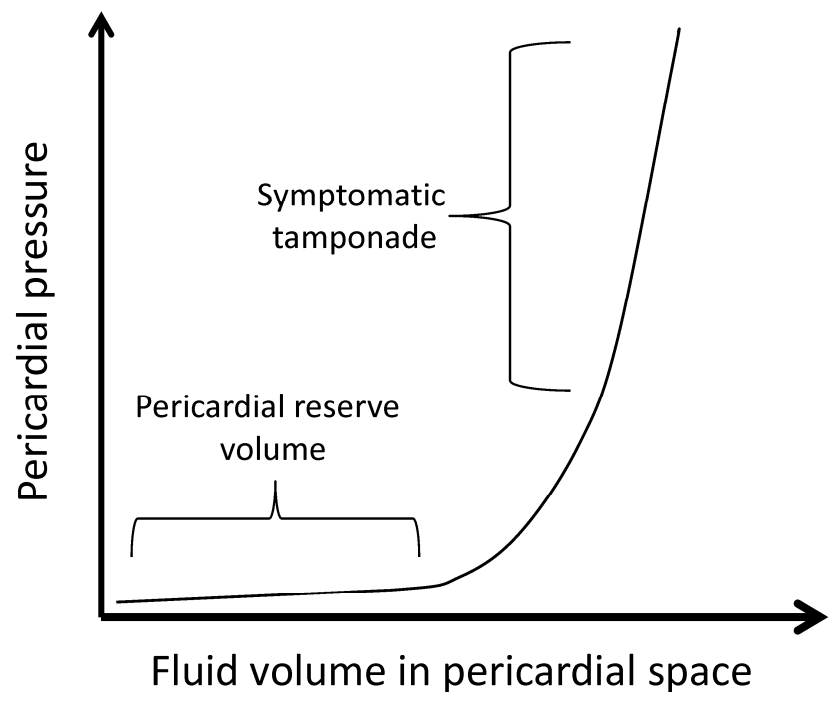

Figure 3. Percardial pressure vs. pericardial volume. As pericardial fluid volume increases, the pericardial reserve volume is filled. Once the reserve volume is full, pressure within the pericardium rapidly rises causing cardiac tamponade and functional depression.

\subsection{The basic physiology associated with the pericardium}

The fibrous (parietal) pericardium is $1-3 \mathrm{~mm}$ thick in healthy humans, and as noted above, is considered as minimally or non-compliant. In fact, because of these features, multiple bioartificial replacement heart valves are made with leaflets of either bovine of swine 
pericardium. As such, this tough layer has the primary function to physically constrain the heart. While this may not have a large influence at rest, during physical exertion, cardiac filling becomes limited by the pericardium. Further, it has been noted that an intact pericardium also increases cardiac chamber interdependence, i.e., increased pressure in one chamber affects other chambers because the total volume is restricted by the pericardium. A more detailed review can be found in the Handbook of Cardiac Anatomy, Physiology, and Devices [50].

Typically during cardiothoracic surgery, the pericardium needs to be opened to obtain direct myocardial access, and at the end of a procedure the pericardium is not typically closed. This in turn reduces the risk of post-surgical cardiac tamponade, as pressure cannot build up easily in an open pericardium. However, the lack of a barrier between the heart and the healing incision site typically leads to scarring and epicardial fusions within this wound site. Typically, this has minor consequences-that is until a subsequent open-heart procedure needs to be performed - and both the initial incisions and heart access are complicated by these additional fibroses. It has been suggested that a barrier placed between the sternum and myocardium would potentially limit the buildup of subsequent adhesions and make reentry less risky. While synthetic barriers such as the absorbable CovaCard (BIOM'UP, Lyon, France) are being developed [51], the native or graft pericardium also may provide a natural and available option.

Relative to physiological consequences, in addition to reducing reoperative complications, the closure of the pericardial sac following cardiac surgery has been proposed to reduce long-term cardiac performance and aid in maintaining diastolic function and ventricular geometry, as well as reduce right ventricular dysfunction [52], [53]. Additionally, one could also consider that a closed pericardium may also provide a reservoir space for subsequent pericardial therapies. Yet, one reported limitation to pericardial closure is that it can acutely reduce cardiac indices and stroke work [54]. More specifically, Rao et al. corroborated that these functions were reduced one hour post-operatively in patients who had pericardial closure $(\mathrm{P}<0.001)$. However, they also reported no significant differences in function between patient groups at 4 hours or 8 hours post-operatively. While increased risk of cardiac tamponade still exists with full closure, it has been reported that fenestrated techniques and pericardial drainage tubes have been used to mitigate the consequences of pericardial effusions [55], [56].

In summary, the pericardial space potentially provides a natural barrier and is well suited for localized drug delivery. Thus if pericardial access could be easily and reproducibly obtained, the possibilities of long- and short-term treatment include: antiarrhythmic therapies, delivery of agents to reduce cellular injuries at reperfusion, and/or use of angiogenic proteins to promote revascularization and regrowth specifically within infarcted regions.

\subsection{Cardioprotective agents}

To date within the US, ischemic heart disease and myocardial infarctions remain as leading causes of clinical morbidity and mortality. Their occurrence often leads to congestive heart 
failure, which in turn causes further reductions in coronary flow and necrosis in the myocardium, and leads to further functional impairments. It is generally considered that compared to other body tissues, myocardial cells have poor regenerative abilities, a fact that has focused much research on methods for reducing trauma and/or myocardial death, as well as methods for improving repair and regeneration.

It has been reported that the local infusion of nitric oxide donors could promote local vasodilation without major systemic effects [57]. Additionally, the administration of VEGF and other angiogenic agents into the myocardium have been associated with several benefits that include: increased collateral vessel development, increases in regional myocardial blood flow, improved myocardial function in the ischemic regions, and/or increased myocardial vascularity [31], [58-60].

Relative to cardioprotective agents, our laboratory has observed that intrapericardial delivery of omega-3 polyunsaturated fatty acids can dramatically reduce both infarct sizes and ventricular arrhythmias associated with ischemia. More specifically in this study, acute ischemia was induced for 45 minutes followed by 180 minutes of reperfusion, while the omega-3 fatty acid docosahexaenoic acid (DHA) was delivered to the pericardial space prior to ischemia as well as during the initial period of reperfusion. Importantly during the ischemic period, ventricular arrhythmias were reduced 50\% (which in the control hearts required defibrillation and caused $20 \%$ mortality). Upon completion of the reperfusion, the hearts were excised and ischemic damage was measured; hearts treated with DHA had a similar area at risk, but a 57\% reduction in normalized infarct size was seen [61]. Ongoing research in our lab also suggests that omega-3 fatty acids may reduce susceptibility to AF during cardiac surgery.

Most recently, investigations in our laboratory have explored the pericardial delivery of specified bile acids noted to have anti-apoptotic benefits. These molecules are upregulated within hibernating black bears, and reports by others have suggested that ursodeoxycholic acid may be beneficial in reducing AF within myocytes [62]. In these ongoing studies to determine potential beneficial effects within a large animal model, we specifically deliver a taurine conjugate of ursodeoxycholic acid within a formed pericardial cradle (the pericardial space) and periodically induce AF. Preliminary results have suggested that these molecules are effective in reducing the times a given heart will elicit AF, i.e., without having to give this therapy intravenously and thus potentially have undesirable systemic side effects (unpublished data).

\subsection{Antiarrhythmic agents}

Antiarrhythmic drugs are commonly known for their narrow therapeutic ranges and severe side effects. It has been previously suggested that delivery of these agents to the pericardial space would allow for myocardial diffusion while lowering undesired plasma drug concentrations [63], [64]. In other words, such local delivery allows for higher doses of this class of agents to be safely administered to control focally the heart rhythm - an application that could be especially applied during cardiothoracic surgery. To date, the intrapericardial 
delivery of antiarrhythmic agents has been attempted with numerous agents, e.g., esmolol [63], solatol [65], atenolol [65], ibutalide [66], procainamide [67], [68], digoxin [67], amiodarone [69], arachadonic acid [70], nitroglycerin [57] and L-arginine [71] have all been shown to have electrophysiological effects when delivered to the pericardial space in various animal models. Additionally, in those studies that also measured plasma concentrations, there was minimal crossover of the delivered agent into the bloodstream [67-69]

To date, despite these reported successes in treating arrhythmias in various animal models, the clinical practice of intrapericardial (IP) delivery is not widely employed. Possible reasons include: 1) the lack of experience (no large clinical trials), 2) difficulties with access and removal of agents, and/or 3) unknown potential complications with this delivery route. It is important to note that one of the described major concerns with pericardial delivery is that it relies primarily on trans-epicardial diffusion to reach the myocardium. In other words, while the thin atria and superficial sinoatrial node may be easy to treat via these mechanisms, the effects of possible ventricular drug gradients are not well defined. Further, it has been hypothesized that moderately soluble or lipophilic molecules will not be evenly transported across the thicker ventricles, causing various degrees of electrophysiological changes through the depth of the myocardium, creating a scenario where the epicardium and endocardium are not conducting and/or contracting at similar rates. This electrical heterogeneity theoretically carries the possibility of initiating, rather than inhibiting, arrhythmias [68].

In our lab, we have investigated the pharmacokinetics and pharmacodynamics of IV and IP delivery of metoprolol [7]. While the $\beta$-blocker is typically used to treat angina and hypertension, it is also used to treat tachycardias. With a tachycardic swine model, IV delivery of metoprolol was faster acting compared to IP, but only by several minutes. While the reductions in heart rates were similar for both delivery techniques, these effects were sustained longer after IP delivery. Importantly, IV delivery was accompanied by significant reductions in contractility, while IP delivery elicited minimal effects. In other words, these findings indicated that IP delivery of metoprolol may have similar bradycardic effects compared to IV delivery, but without the reduced contractility. The other important finding in this study was the minimal pericardial crossover of metoprolol within blood, as well as the slightly increased half-life of the drug [7].

\subsection{Clinical pericardial access}

Access to the pericardial space for the delivery of therapies outside of cardiothoracic surgical procedures may pose many difficulties. However, multiple minimally invasive procedural methodologies are under development. For example in the trans-atrial approach, access to the pericardium is achieved via a catheter coming up through the femoral vein into the right atrial appendage, where it then punctures through this thin myocardium to gain access into the pericardial space. To date, success with this approach has been demonstrated in canines and swine [72]. Alternatively, a subxyphoid access procedure has been suggested 
[73]. While this method is often used to drain the pericardial space during episodes of cardiac tamponade, the minimal separation between the fibrous pericardium and the epicardium make this approach more difficult when there is not a substantial amount of intrapericardial fluid present. Several access tools have and continue to be developed to aid in subxyphoid access using minimally invasive approaches. For instance, the PeriPort ${ }^{\mathrm{TM}}$ (Cormedics, TX) system is designed to initially enter the thoracic space through a subxyphoid incision, where it uses a vacuum on the distal end to grip the pericardium and separate it from the heart's epicardial surface. Once the pericardium is pulled into the vacuum chamber, a retractable needle pierces the pericardium and allows for a near tangential entrance of a guidewire. Such designed access tools should enhance the safety and simplicity of pericardial access and also have potential to increase the widespread use of localized therapies to treat pericardial and cardiac diseases. Nevertheless, during cardiothoracic surgery, a simple syringe or perfusion pump is all that is clinically needed to deliver pericardial therapies. Thus the hurdles to add localized pericardial delivery of drugs concurrent to surgery are much less compared to pericardial therapies on their own.

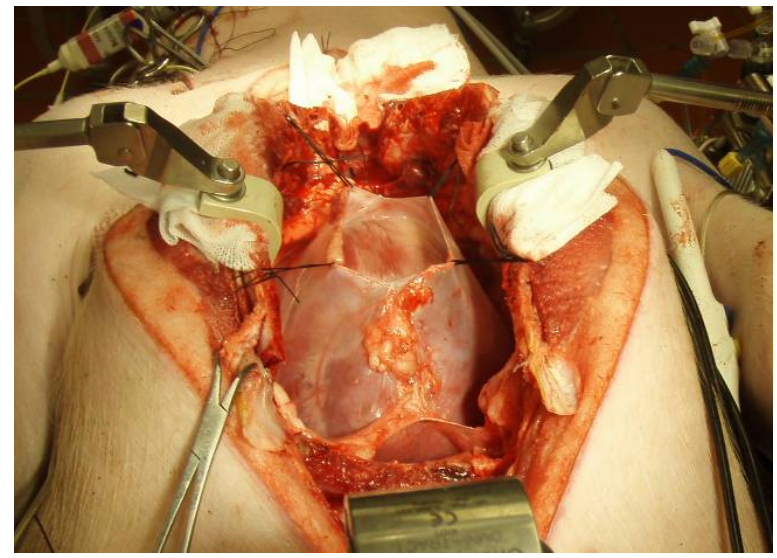

Figure 4. Methods of pericardial access include the pericardium as a reservoir. Pictured here is a pericardial cradle in a swine model.

\subsection{Protocols}

As noted above, the pericardial delivery of molecules, cells, nanoparticles, etc., becomes simplified with surgical access (Figure 4). Without the requirement of added procedures and incisions, the pericardial delivery of therapeutics can be administered with minimal additional equipment, time, or complications, leading to numerous clinical scenarios that may benefit from the use of pericardial delivery. While some of these applications, as described below, have been tested in pilot studies in animals, future clinical studies have yet to be developed to confirm efficacy.

In one such application, a common first choice of donor vessel for a CABG procedure is the internal thoracic artery. Thus, while the surgeon initially frees and prepares this vessel for 
subsequent grafting, the pericardial administration of either a prophylactic antiarrhythmic or angiogenic agent could be infused into the pericardium. Once the artery has been prepared and the pericardium is further opened, the drug has a chance to partially diffuse into the myocardial tissue. Additional drugs could also be added through direct myocardial injection to promote revascularization or prevent post-operative arrhythmias. Alternatively, a procedure that requires access from the anterior surface of the heart may make use of the reservoir created by the remaining pericardium to treat the posterior myocardium during the procedure.

Another important approach to consider is the post-operative administration of drugs, which could be accomplished via surgically placed pericardial drainage catheters. More specifically, with the placement of pleural drainage catheters and temporary pacing leads at the end of a procedure, the addition of a catheter leading into the pericardium is sometimes included [74]. This catheter, whether through the main lumen or a second lumen, could hypothetically allow for continued access to the pericardial space and delivery of appropriate antiarrhythmic, antibiotic, or other therapies to the myocardium. Therapies with such devices have been attempted with success in a porcine model [75].

As mentioned above, multiple techniques exist for low or no tension pericardial closures [56], [76], [77]. In addition to reoperative benefits, the planned closure of the pericardium can create a useful reservoir for localized treatment. While the most common clinical reason for non-closure is to reduce the risk of cardiac tamponade, this could be mitigated with the placement of the aforementioned specially designed drainage/therapy delivery catheter. We also believe that with full pericardial closure and access via the pericardial drain, a delivered therapy would reach all surfaces of the heart.

Another clinical situation/option for localized drug delivery might be during cardiopulmonary bypass procedures. To arrest the heart and often throughout the procedure, cardioplegia solutions are perfused through the coronary vessels. Whether antiegrade or retrograde, this perfused solution cools and protects the heart by minimizing metabolism. It has been considered that myocardial damage can be further reduced by introducing cardioprotective agents via this delivery route [78-80]. It is important to note that in this unique situation, any administered drug reaches the entire myocardium via the capillaries, but will have no access to other tissues until after cross clamping is released. In other words, high transmural and widespread myocardial concentrations can be achieved with remarkable speed and accuracy while minimizing side effects.

Similarly, organ transplantation offers ultimate accessibility in localized drug delivery. A typical transplant, includes explant, transportation and re-implantation. Because of the recovery of multiple organs, localized delivery is a viable option where the effects can be greatly enhanced after re-implantation function. Not only are options such as pericardial delivery still available since the heart is typically the last organ removed, but IV therapies just prior to cross-clamping become targeted. 
Next, the times between explant and implant can range from under six hours for the heart to up to 24 hours for the liver or kidney. During these times, the heart is often in stagnant cold storage. However, ideas and methods for continuous perfusion during the hypothermic period are well established, e.g., continuous hypothermic perfusion of the heart was first applied only a year after the first successful human heart transplant [81]. Since then it shown that it is possible to keep large mammalian hearts viable for up to 48 hours in baboons and swine [82], as well as improve function compared to non-perfused hearts [83]. While continuous hypothermic perfusion is not used clinically with heart transplantation, it should be noted that the Organ Care System (Transmedics, Andover, MA) gained investigational device exemption status in 2007, and a clinical trial is currently underway. This continuous perfusion during transport before implant provides another opportunity for localized therapies, as they could be simply added to the perfusates.

\section{Limitations and conclusions}

Localized delivery methods such as those into the pericardial space are not without potential limitations. Without direct access to the bloodstream, an administered agent at a target tissue, such as in the pericardial space, must diffuse into the target tissue to increase concentrations. While this may ultimately limit the depth or distance a given therapeutic agent (drugs, cells, nanoparticles, etc.) might be able to migrate in a significant quantity, it is also one of the major advantages of localized therapies.

Some areas for localized treatments, especially within the myocardium, may be considered as relatively difficult to access. However, with a large number of cardiac surgeries currently being performed and an increasing number of catheter procedures being developed and implemented, these therapeutic methods could piggyback on planned procedures with few added complications or risks. Finally, novel therapeutic deliveries, drugs, or other interventions may need to gain additional approval in order to become indications for localized delivery.

Cardiothoracic surgeries may facilitate novel opportunities for local drug delivery, such as overcoming the hurdle to obtaining pericardial access. Such clinical procedures also provide opportunities for localized injections or coronary infusions. The future opportunities for such therapies are not limited to the duration of the operation; by leaving a pericardial drainage catheter with delivery features, physicians could also incorporate subsequent therapeutic delivery. In some cases, implantable drug pumps or biodegradable patches could provide therapy to the patient beyond their ICU stay. The emerging field of localized therapy delivery shows great potential, but future human studies are needed to verify the positive results observed in pre-clinical studies. Nevertheless, the unique access afforded by cardiothoracic procedures may speed up implementation of these promising local and target therapeutic delivery methods, thus placing surgeons at the cutting edge of novel delivery approaches. 


\section{Author details}

Christopher Rolfes, Stephen Howard and Ryan Goff

Departments of Biomedical Engineering and Surgery, University of Minnesota, Minneapolis, MN, USA

Paul A. Iaizzo*

Departments of Biomedical Engineering, Surgery, Integrative Biology and Physiology, University of Minnesota, Minneapolis, MN, USA

\section{References}

[1] Center for Drug Evaluation and Research and Food and Drug Administration, "Guidance for Industry: Bioavailability and Bioequivalence Studies for Orally Administered Drug Products - General Considerations," 2003.

[2] S. P. Jones et al., "Leukocyte and endothelial cell adhesion molecules in a chronic murine model of myocardial reperfusion injury Leukocyte and endothelial cell adhesion molecules in a chronic murine model of myocardial reperfusion injury," Group, 2012.

[3] B. J. Lestini et al., "Surface modification of liposomes for selective cell targeting in cardiovascular drug delivery," Journal of Controlled Release, vol. 78, no. 1-3, pp. 235-47, Jan. 2002.

[4] J. R. Lindner and S. Kaul, "Delivery of drugs with ultrasound," Echocardiography (Mount Kisco, N.Y.), vol. 18, no. 4, pp. 329-37, May 2001.

[5] C. M. H. Newman and T. Bettinger, "Gene therapy progress and prospects: ultrasound for gene transfer," Gene Therapy, vol. 14, no. 6, pp. 465-75, Mar. 2007.

[6] D. Vancraeynest et al., "Myocardial delivery of colloid nanoparticles using ultrasoundtargeted microbubble destruction," European Heart Journal, vol. 27, no. 2, pp. 237-45, Jan. 2006.

[7] E. S. Richardson, C. Rolfes, O. Woo, W. Elmquist, D. Benditt, and P. A. Iaizzo, “Cardiac response to the intrapericardial delivery of metoprolol: targeted delivery compared to intravenous administration," Journal of Cardiovascular Translational Research, vol. 5, no.4, pp535-40, Aug. 2012.

[8] G. M. McKhann, M. A. Grega, L. M. Borowicz, W. A. Baumgartner, and O. A. Selnes, "Stroke and encephalopathy after cardiac surgery," Stroke, vol. 37, no. 2, pp. 562-71, Feb. 2006.

[9] A. C. Breuer et al., "Central nervous system complications of coronary artery bypass graft surgery: prospective analysis of 421 patients," Stroke, vol. 14, no. 5, pp. 682-87, Oct. 1983.

[10] D. Barbut and L. R. Caplan, "Brain complications of cardiac surgery," Current Problems in Cardiology, vol. 22, no. 9, pp. 449-80, Sep. 1997.

${ }^{*}$ Corresponding Author 
[11] A. J. Furlan and A. C. Breuer, “Central nervous system complications of open heart surgery," Stroke, vol. 15, no. 5, pp. 912-15, Oct. 1984.

[12] E. E. Tseng et al., "Neuronal nitric oxide synthase inhibition reduces neuronal apoptosis after hypothermic circulatory arrest," The Annals of Thoracic Surgery, vol. 64, no. 6, pp. 1639-47, Dec. 1997.

[13] E. E. Tseng, M. V. Brock, and C. C. Kwon, "Quantitative analyses of intracerebral excitatory amino acids and citulline following hypothermic circulatory arrest," Surgical Forum, vol. 48, pp. 297-9, 1997.

[14] D. C. Harmon, K. G. Ghori, N. P. Eustace, S. J. F. O'Callaghan, A. P. O’Donnell, and G. D. Shorten, "Aprotinin decreases the incidence of cognitive deficit following $\{C A B G\}$ and cardiopulmonary bypass: a pilot randomized controlled study," Canadian Journal of Anaesthesia, vol. 51, no. 10, pp. 1002-9, Dec. 2004.

[15] J. P. Mathew et al., "A multicenter risk index for atrial fibrillation after cardiac surgery," The Journal of the American Medical Association, vol. 291, no. 14, pp. 1720-9, Apr. 2004.

[16] C. V. Egleston, A. E. Wood, T. F. Gorey, and E. M. McGovern, “Gastrointestinal complications after cardiac surgery," Annals of The Royal College of Surgeons of England, vol. 75, no. 1, pp. 52-6, Jan. 1993.

[17] B. Andersson, J. Nilsson, J. Brandt, P. Höglund, and R. Andersson, “Gastrointestinal complications after cardiac surgery," British Journal of Surgery, vol. 92, no. 3, pp. 326-33, Mar. 2005.

[18] G. M. Sreeram, I. J. Welsby, A. D. Sharma, B. Phillips-Bute, P. K. Smith, and T. F. Slaughter, "Infectious complications after cardiac surgery: lack of association with fresh frozen plasma or platelet transfusions," Journal of Cardiothoracic and Vascular Anesthesia, vol. 19, no. 4, pp. 430-4, Aug. 2005.

[19] M. C. Engoren, R. H. Habib, A. Zacharias, T. A. Schwann, C. J. Riordan, and S. J. Durham, "Effect of blood transfusion on long-term survival after cardiac operation," The Annals of Thoracic Surgery, vol. 74, no. 4, pp. 1180-6, Oct. 2002.

[20] B. D. Spiess, "Transfusion of blood products affects outcome in cardiac surgery," Seminars in Cardiothoracic and Vascular Anesthesia, vol. 8, no. 4, pp. 267-81, Dec. 2004.

[21] J. F. Rey et al., "Feasibility of stomach exploration with a guided capsule endoscope," Endoscopy, vol. 42, no. 7, pp. 541-5, Jul. 2010.

[22] G. Kósa, P. Jakab, G. Székely, and N. Hata, “\{MRI\} driven magnetic microswimmers," Biomedical Microdevices, vol. 14, no. 1, pp. 165-78, Feb. 2012.

[23] B. Laulicht, N. J. Gidmark, A. Tripathi, and E. Mathiowitz, "Localization of magnetic pills," Proceedings of the National Academy of Sciences of the United States of America, vol. 108, no. 6, pp. 2252-7, Feb. 2011.

[24] R. Hovorka et al., “Overnight closed loop insulin delivery (artificial pancreas) in adults with type 1 diabetes: crossover randomised controlled studies," British Medical Journal, vol. 342, p. d1855-d1855, Apr. 2011. 
[25] R. Hovorka et al., "Manual closed-loop insulin delivery in children and adolescents with type 1 diabetes: a phase 2 randomised crossover trial," Lancet, vol. 375, no. 9716, pp. 743-51, Feb. 2010.

[26] S. Weinzimer, G. Steil, K. Swan, J. Dziura, N. Kurtz, and W. Tamborlane, "Fully automated closed-loop insulin delivery versus semiautomated hybrid control in pediatric patients with type 1 diabetes using an artificial pancreas," Diabetes Care, vol. 31, pp. 934-9, 2008.

[27] E. Renard, "Implantable closed-loop glucose-sensing and insulin delivery: the future for insulin pump therapy," Current Opinion in Pharmacology, vol. 2, no. 6, pp. 708-16, Dec. 2002.

[28] S. Okubo, O. Wildner, M. R. Shah, J. C. Chelliah, M. L. Hess, and R. C. Kukreja, “Gene transfer of heat-shock protein 70 reduces infarct," In Vivo, pp. 877-881, 2001.

[29] V. Jayasankar et al., "Targeted overexpression of growth hormone by adenoviral gene transfer preserves myocardial function and ventricular geometry in ischemic cardiomyopathy," Journal of Molecular and Cellular Cardiology, vol. 36, no. 4, pp. 531-8, Apr. 2004.

[30] V. Jayasankar, L. T. Bish, T. J. Pirolli, M. F. Berry, J. Burdick, and Y. J. Woo, “Local myocardial overexpression of growth hormone attenuates postinfarction remodeling and preserves cardiac function," The Annals of Thoracic Surgery, vol. 77, no. 6, pp. 2122-9; discussion 2129, Jun. 2004.

[31] D. W. Losordo et al., "Gene therapy for myocardial angiogenesis," Online, pp. 2800-4, 1998.

[32] N. M. Elman, Y. Patta, A. W. Scott, B. Masi, H. L. Ho Duc, and M. J. Cima, "The next generation of drug-delivery microdevices," Clinical Pharmacology and Therapeutics, vol. 85, no. 5, pp. 544-7, May 2009.

[33] R. De Souza, P. Zahedi, C. J. Allen, and M. Piquette-Miller, "Polymeric drug delivery systems for localized cancer chemotherapy," Drug Delivery, vol. 17, no. 6, pp. 365-75, Aug. 2010.

[34] J. Leor, Y. Amsalem, and S. Cohen, “Cells, scaffolds, and molecules for myocardial tissue engineering," Pharmacology \& Therapeutics, vol. 105, no. 2, pp. 151-63, Feb. 2005.

[35] D. Brieger and E. Topol, "Local drug delivery systems and prevention of restenosis," Cardiovascular Research, vol. 35, no. 3, pp. 405-13, Sep. 1997.

[36] P. Barath, A. Popov, G. L. Dillehay, G. Matos, and T. McKiernan, "Infiltrator angioplasty balloon catheter: a device for combined angioplasty and intramural sitespecific treatment," Catheterization and Cardiovascular Diagnosis, vol. 41, no. 3, pp. 333-41, Jul. 1997.

[37] B. Scheller et al., "Treatment of coronary in-stent restenosis with a paclitaxel-coated balloon catheter," New England Journal of Medicine, vol. 355, pp. 2113-24, 2006.

[38] L. A. Guzman et al., "Local intraluminal infusion of biodegradable polymeric nanoparticles," Heart, pp. 1441-8, 1996. 
[39] R. Langer, "New methods of drug delivery," Science, vol. 249, no. 4976, pp. 1527-33, Sep. 1990.

[40] J. Kost, T. Horbett, B. Ratner, and M. Singh, "Glucose-sensitive membranes containing glucose oxidase: activity, swelling, and permeability studies," Journal of Biomedical Materials Research, vol. 19, no. 9, pp. 1117-33, Nov-Dec. 1985.

[41] A. Michael, E. Buffen, R. Rauck, W. Anderson, M. McGirt, and H. V. Mendenhall, “An in vivo canine study to assess granulomatous responses in the MedStream Programmable Infusion System (TM) and the SynchroMed II Infusion System ${ }^{\circledR}, "$ Pain Medicine, vol. 13, no. 2, pp. 175-84, Feb. 2012.

[42] Y. H. Bae and K. Park, "Targeted drug delivery to tumors: myths, reality and possibility," Journal of Controlled Release, vol. 153, no. 3, pp. 198-205, Aug. 2011.

[43] R. J. Price, D. M. Skyba, S. Kaul, and T. C. Skalak, “Delivery of colloidal particles and red blood cells to tissue through microvessel ruptures created by targeted microbubble destruction with ultrasound," Online, pp. 1264-7, 1998.

[44] T. Ay et al., "Destruction of contrast microbubbles by ultrasound: effects on myocardial function, coronary perfusion pressure, and microvascular integrity," Circulation, vol. 104, no. 4, pp. 461-6, Jul. 2001.

[45] D. E. Meyer, B. C. Shin, G. a Kong, M. W. Dewhirst, and A. Chilkoti, "Drug targeting using thermally responsive polymers and local hyperthermia," Journal of Controlled Release, vol. 74, no. 1-3, pp. 213-24, Jul. 2001.

[46] A. Gibson and M. B. Segal, "A study of the composition of pericardial fluid, with special reference to the probably mechanism of fluid formation," Journal of Physiology, vol. 277, pp. 367-77, 1978.

[47] B. Boulanger, Z. Yuan, M. Flessner, J. Hay, and M. Johnston, "Pericardial fluid absorption into lymphatic vessels in sheep," Microvascular Research, vol. 57, no. 2, pp. 174-86, Mar. 1999.

[48] Z. Yuan, B. Boulanger, M. Flessner, and M. Johnston, "Relationship between pericardial pressure and lymphatic pericardial fluid transport in sheep," Microvascular Research, vol. 60, no. 1, pp. 28-36, Jul. 2000.

[49] B. L. Pegram and V. S. Bishop, "An evaluation of the pericardial sac as a safety factor during tamponade," Cardiovascular Research, vol. 9, pp. 715-21, 1975.

[50] E. S. Richardson, A. J. Hill, N. D. Skadsberg, M. R. Ujhelyi, Y.-F. Xiao, and P. A. Iaizzo, "The Pericardium," in Handbook of Cardiac Anatomy, Physiology and Devices, second edition, 2009, pp. 125-36.

[51] A. Bel et al., "Prevention of postcardiopulmonary bypass pericardial adhesions by a new resorbable collagen membrane," Interactive Cardiovascular and Thoracic Surgery, vol. 0, pp. 1-5, Jan. 2012.

[52] R. Shabetai, The Pericardium. 2003, pp. 1-85.

[53] M. Watkins and M. LeWinter, "Physiologic role of the normal pericardium," Annual Review Medicine, vol. 44, pp. 171-80, 1993. 
[54] G. D. Angelini et al., "Adverse hemodynamic effects and echocardiographic consequences of pericardial closure soon after sternotomy and pericardiotomy," Circulation, vol. 82, no. 5, pp. IV397-406, Nov. 1990.

[55] H. Ekim, V. Kutay, A. Hazar, H. Akbayrak, H. Basel, and M. Tuncer, "Effects of posterior pericardiotomy on the incidence of pericardial effusion and atrial fibrillation after coronary revascularization," Medical Science Monitor, vol. 12, no. 10, pp. 431-4, 2006.

[56] F. Kargar and M. H. Aazami, "Rotational pericardial flap: an alternative tension-free technique for pericardial closure," The Journal of Thoracic and Cardiovascular Surgery, vol. 134, no. 2, pp. 510-1, Aug. 2007.

[57] K. Kumar et al., "Potent antifibrillatory effects of intrapericardial nitroglycerin in the ischemic porcine heart," Journal of the American College of Cardiology, vol. 41, no. 10, pp. 1831-7, May 2003.

[58] R. J. Laham, M. Rezaee, M. Post, X. Xu, and F. W. Sellke, “Intrapericardial administration of basic fibroblast growth factor: myocardial and tissue distribution and comparison with intracoronary and intravenous administration," Catheterization and Cardiovascular Interventions, vol. 58, no. 3, pp. 375-81, Mar. 2003.

[59] D. F. Lazarous et al., "Pharmacodynamics of basic fibroblast growth factor: route of administration determines myocardial and systemic distribution," Cardiovascular Research, vol. 36, no. 1, pp. 78-85, Oct. 1997.

[60] R. A. Tio, J. G. Grandjean, A. J. H. Suurmeijer, W. H. van Gilst, D. J. van Veldhuisen, and A. J. van Boven, "Thoracoscopic monitoring for pericardial application of local drug or gene therapy," International Journal of Cardiology, vol. 82, no. 2, pp. 117-21, Feb. 2002.

[61] Y.-F. Xiao, D. C. Sigg, M. R. Ujhelyi, J. J. Wilhelm, E. S. Richardson, and P. A. Iaizzo, "Pericardial delivery of omega-3 fatty acid: a novel approach to reducing myocardial infarct sizes and arrhythmias," American Journal of Physiology. Heart and Circulatory Physiology, vol. 294, no. 5, pp. H2212-8, May 2008.

[62] M. Miragoli et al., "A protective antiarrhythmic role of ursodeoxycholic acid in an in vitro rat model of the cholestatic fetal heart," Hepatology, vol. 54, no. 4, pp. 1282-92, Oct. 2011.

[63] R. Moreno, S. Waxman, K. Rowe, R. Verrier, and C. von Schacky, "Intrapericardial [beta]-adrenergic blockade with esmolol exerts a potent antitachycardic effect without depressing contractility," Journal of Cardiovascular Pharmacology, vol. 36, pp. 722-7, 2000.

[64] T. J. van Brakel et al., "Intrapericardial delivery enhances cardiac effects of sotalol and atenolol," Journal of Cardiovascular Pharmacology, vol. 44, no. 1, pp. 50-6, Jul. 2004.

[65] T. J. van Brakel et al., "Intrapericardial delivery enhances cardiac effects of sotalol and atenolol," Journal of Cardiovascular Pharmacology, vol. 44, no. 1, pp. 50-6, Jul. 2004.

[66] A. Vereckei, J. C. Gorski, M. Ujhelyi, R. Mehra, and D. P. Zipes, "Intrapericardial ibutilide administration fails to terminate pacing-induced sustained atrial fibrillation in dogs," Cardiovascular Drugs and, vol. 18, no. 4, pp. 269-77, Jul. 2004. 
[67] T. M. Kolettis et al., "Intrapericardial drug delivery: pharmacologic properties and long-term safety in swine," International Journal of Cardiology, vol. 99, no. 3, pp. 415-21, Mar. 2005.

[68] M. R. Ujhelyi, K. Z. Hadsall, D. E. Euler, and R. Mehra, "Intrapericardial therapeutics: a pharmacodynamic and pharmacokinetic comparison between pericardial and intravenous procainamide delivery," Journal of Cardiovascular Electrophysiology, vol. 13, no. 6, pp. 605-11, Jun. 2002.

[69] G. M. Ayers, T. H. Rho, J. Ben-David, H. R. Besch, and D. P. Zipes, “Amiodarone instilled into the canine pericardial sac migrates transmurally to produce electrophysiologic effects and suppress atrial fibrillation," Journal of Cardiovascular Electrophysiology, vol. 7, no. 8, pp. 713-21, Aug. 1996.

[70] T. Miyazaki and D. P. Zipes, "Pencardial prostaglandin biosynthesis prevents the increased incidence of reperfusion-induced ventricular fibrillation produced by efferent sympathetic stimulation in dogs," Online, pp. 1008-19, 1990.

[71] L. Fei, A. Baron, D. Henry, and D. P. Zipes, "Intrapericardial delivery of L-arginine reduces the increased severity of ventricular arrhythmias during sympathetic stimulation in dogs with acute coronary occlusion," Circulation, vol. 96, pp. 4044-9, 1997.

[72] R. L. Verrier, S. Waxman, E. G. Lovett, and R. Moreno, "Transatrial access to the normal pericardial space and therapeutic interventions," Online, pp. 2331-3, 1998.

[73] A. P. Mannam "Safety of subxyphoid pericardial access using a blunt-tip needle," American Journal of Cardiology, vol. 89, no. 7, pp. 891-3, 2002.

[74] S. Eryilmaz et al., "Effect of posterior pericardial drainage on the incidence of pericardial effusion after ascending aortic surgery," The Journal of Thoracic and Cardiovascular Surgery, vol. 132, no. 1, pp. 27-31, Jul. 2006.

[75] E. S. Richardson, B. Whitson, and P. Iaizzo, "A novel combination therapy for postoperative arrhythmias," Journal of Medical Devices, vol. 3, no. 2, p. 27511, 2009.

[76] V. Rao, M. Komeda, R. D. Weisel, G. Cohen, M. A. Borger, and T. E. David, "Should the pericardium be closed routinely after heart operations?," The Annals of Thoracic Surgery, vol. 67, no. 2, pp. 484-8, Feb. 1999.

[77] G. Bhatnagar, S. E. Fremes, G. T. Christakis, and B. S. Goldman, "Early results using an ePTFE membrane for pericardial closure following coronary bypass grafting," Journal of Cardiac Surgery, vol. 13, no. 3, pp. 190-3, May 1998.

[78] H. Hwang et al., "Ranolazine as a cardioplegia additive improves recovery of diastolic function in isolated rat hearts," Circulation, vol. 120, no. 11, pp. S16-21, Sep. 2009.

[79] R. M. Mentzer et al., "Adenosine as an additive to blood cardioplegia in humans during coronary artery bypass surgery," Society, pp. 38-43, 1997.

[80] J. A. Coles, D. C. Sigg, and P. A. Iaizzo, "The potential benefits of $1.5 \%$ hetastarch as a cardioplegia additive," Biochemical Pharmacology, vol. 69, no. 11, pp. 1553-8, Jun. 2005.

[81] E. Proctor and R. Parker, "Preservation of isolated heart for 72 Hours," British Medical Journal, vol. 4, no. 2, pp. 296-8, Jan. 1968. 
[82] W. N. Wicomb, D. Novitzky, D. Cooper, and A. Rose, "Forty-eight hours hypothermic perfusion storage of pig and baboon hearts," Journal of Surgical Research, vol. 40, pp. 27684,1986

[83] T. Ozeki et al., "Heart preservation using continuous ex vivo perfusion improves viability and functional recovery," Circulation journal, vol. 71, pp. 153-9, 2007. 